\title{
POLYMORPHISM OF A MYCOBACTERIAL ANTIGEN PARTICIPATING IN CELL-MEDIATED IMMUNITY
}

\author{
Sandhya Hattikudur and R. S. Kamat \\ Department of Immunology, Haffkine Institute, Parel, Bombay 400 012, India
}

\begin{abstract}
SUMmaRY. The cross-reactivity of crude sonic extracts of six species of mycobacteria was studied by skin tests in mice and guinea pigs immunised with live Mycobacterium tuberculosis strain H37Rv, and in patients with pulmonary tuberculosis. All slowly growing mycobacteria elicited a strong delayed hypersensitivity response, $M$. vaccae a moderate response and $M$. phlei a poor or no response. A specific target antigen for cell-mediated immunity, known to be present in $M$. tuberculosis, was also present in all the mycobacteria studied. This shared antigen was shown, by immunoprecipitation tests, to be identical in all the slowly growing species but only a partial reaction of identity was obtained with $M$. phlei. It is concluded that the antigen shared by the slowly growing mycobacteria is immunodominant in cell-mediated immunity.
\end{abstract}

\section{INTRODUCTION}

Knowledge of the antigenic relationships amongst mycobacteria is important because of epidemiological and other implications. An individual may acquire immunity to a particular pathogen as a result of infection with antigenically related environmental mycobacteria of low virulence. This has been suggested as one of the reasons for the failure of some BCG vaccination programmes (WHO report, 1979).

In a recent publication (Hattikudur and Kamat, 1984) we reported the isolation and characterisation of a protein antigen of Mycobacterium tuberculosis strain $\mathrm{H} 37 \mathrm{Rv}$ that is a target for not only the delayed hypersensitivity response but also for protective cell-mediated immunity against the organism. In this report we present evidence of cross-reactivity between different mycobacteria and $M$. tuberculosis employing delayed hypersensitivity as the test system. We have further analysed the cross-reactivity employing the above mentioned target antigen of cell-mediated immunity as a reference marker.

\section{MATERIAL AND METHODS}

Bacteria. M. tuberculosis strain H37Rv (NCTC7416), M. bovis var. BCG (NCTC5692), $M$. marinum (NCTC2275), M. avium (NCTC8559) and $M$. phlei (NCTC8151) were obtained from the National Collection of Type Cultures, Colindale Avenue, London NW9 5HT. M. vaccae was supplied by Dr J. L. Stanford, Middlesex Hospital, London. 
Preparation of sonic extracts. Strains were grown on the medium of Doub and Youmans (1950) for 2-8 weeks and killed by exposure to $2 \cdot 4$-megarad gamma radiation from a ${ }^{60} \mathrm{Co}$ source. The killed bacterial cell mass was harvested and washed with phosphate buffered saline (PBS, $p \mathrm{H} \mathrm{7.2)} \mathrm{and} \mathrm{disrupted} \mathrm{by} \mathrm{sonic} \mathrm{disintegration} \mathrm{(Sonifier} \mathrm{B} \mathrm{30,} \mathrm{Branson,} \mathrm{USA).} \mathrm{The} \mathrm{sonicate}$ was centrifuged at $50000 \mathrm{~g}$ for $1 \mathrm{~h}$ to remove the cell debris and the supernate stored in 3-ml portions at $-40^{\circ} \mathrm{C}$. Portions were thawed and the protein concentration estimated by the method of Lowry et al. (1951). Tween 80 was added to give a final concentration of $0.01 \%$ before storage at $4^{\circ} \mathrm{C}$.

Reference antigen and antiserum. The antigen capable of eliciting effector responses of cell-mediated immunity against $M$. tuberculosis strain H37Rv was purified as described by Hattikudur and Kamat (1984). The antiserum was raised in rabbits by four injections of $100 \mu \mathrm{g}$ of reference antigen of $M$. tuberculosis strain H37Rv, in Freund's incomplete adjuvant. The antiserum had a titre of 32 against the reference antigen when tested by the gel diffusion technique of Ouchterlony and Nilsson (1978).

Immunoelectrophoretic and agar gel techniques. Two-dimensional immunoelectrophoresis (2D-IEP), rocket immunoelectrophoresis and agar gel diffusion precipitation techniques were performed as described by Hattikudur and Kamat (1984).

Immunisation of animals. Outbred guinea pigs weighing $c .200 \mathrm{~g}$ and Swiss white mice weighing $c .20 \mathrm{~g}$ were purchased from Haffkine Biopharmaceutical Corp. Ltd, Bombay, India. They were immunised with $0.25 \mathrm{mg}$ and $0.1 \mathrm{mg}$ net weight respectively of live cells of $M$. tuberculosis strain H37Rv injected intraperitoneally (ip). The animals were tested for delayed hypersensitivity responses to the sonic extracts of the six strains of mycobacteria prior to immunisation and only the non-reactors were selected.

Delayed hypersensitivity tests were made in the animals immunised with $M$. tuberculosis strain H37Rv and patients suffering from pulmonary tuberculosis proven by demonstration of acid-fast bacilli in their sputa. Only animals that gave a statistically significant delayed hypersensitivity response to sonic extract of strain H37Rv and patients that showed reactions to PPD $>8.0 \mathrm{~mm}$ in diameter were selected as test individuals. In man, 5 tuberculin units of PPD-S (Serum Institute, Denmark) in $0.1 \mathrm{ml}$ of PBS was injected intradermally into the forearm as a positive control. Diameters of erythema and induration were read $24 \mathrm{~h}$ and $48 \mathrm{~h}$ after injection. Test sonic extract $0.1 \mu \mathrm{g}$ in $0.1 \mathrm{ml}$ of PBS was similarly tested in at least five individuals who gave positive delayed hypersensitivity responses $(>8.0 \mathrm{~mm})$ to PPD. In guinea pigs, the abdominal skin was depilated and $30 \mu \mathrm{g}$ of protein of the test sonic extract in $0.1 \mathrm{ml}$ of PBS containing $0.01 \%$ Tween 80 was injected intradermally. The diameter of erythema and induration was measured 24 $\mathrm{h}$ and $48 \mathrm{~h}$ after injection. In each guinea pig, three sonic extracts were tested in addition to the sonic extract of $\boldsymbol{M}$. tuberculosis strain H37Rv. Each sonic extract was tested in five guinea pigs that gave a good delayed hypersensitivity response to $M$. tuberculosis strain $\mathrm{H} 37 \mathrm{Rv}$. In mice, 30 $\mu \mathrm{g}$ of sonic extract in $0.03 \mathrm{ml}$ was injected into the left hind foot pad and measurements were taken before, and 24 and $48 \mathrm{~h}$ after injection (Gray and Jennings, 1955). The sonic extract of each test organism was injected into the right foot pad of at least five mice that gave a positive delayed hypersensitivity response to the sonic extract of $M$. tuberculosis strain H37Rv injected into the left foot pad simultaneously. As controls, delayed hypersensitivity tests were performed with all the sonic extracts in normal non-immune guinea pigs and mice. The diameters of skin reactions and foot pad swelling readings were analysed statistically by Student's $t$ test (Freund 1967) in comparison with corresponding readings in non-immune negative controls.

\section{RESULTS}

\section{Cross-reactivity of different mycobacteria with $M$. tuberculosis strain $H 37 R v$}

Sonicates of $M$. tuberculosis strain H37Rv, BCG, $M$. marinum and $M$. avium showed statistically significant delayed hypersensitivity responses in all the three species. $M$. vaccae, a rapidly growing organism of low virulence in man, showed a significant but lower response, whereas $M$. phlei, a rapidly growing saprophyte, 
TABLE I

Delayed hypersensitivity cross-reactivity of mycobacteria with $M$. tuberculosis strain H37Rv

\begin{tabular}{l|ccc}
\hline & \multicolumn{2}{|c}{$\begin{array}{c}\text { Delayed hypersensitivity } \\
\text { response (mm) } \\
\text { (mean } \pm \text { SEM) }\end{array}$} & $\begin{array}{c}\text { Increase in } \\
\text { mouse footpad } \\
\text { Test }\end{array}$ \\
\cline { 2 - 4 } antigens & Guinea pig & Man & at $48 \mathrm{~h}$ \\
\hline M. tuberculosis & & & \\
strain H37Rv & $19.3 \pm 1.9$ & $14.9 \pm 0.65$ & $0.462 \pm 0.045$ \\
M. avium & $15.5 \pm 1.5$ & $11.6 \pm 1.21$ & $0.2 \pm 0.035$ \\
BCG & $9.0 \pm 1.0$ & $12.6 \pm 0.81$ & $0.183 \pm 0.017$ \\
M. marinum & $8.33 \pm 1.2$ & $11.3 \pm 0.93$ & $0.192 \pm 0.031$ \\
M. vaccae & $7.3 \pm 1.12$ & $8.33 \pm 0.76$ & $0.133 \pm 0.022$ \\
M. phlei & $5.25 \pm 0.63$ & $1.97^{*} \pm 0.87$ & $0.11^{*} \pm 0.024$ \\
\hline
\end{tabular}

The delayed hypersensitivity responses were statistically significant $(p<0.01)$ in comparison with the reactions in non-immune controls.

* These delayed hypersensitivity responses were not significant.

showed practically no response in man, an insignificant response in mice and a very low but significant response in guinea pigs (table I).

Screening of sonic extracts of various mycobacteria for presence of 0.85-Rf antigen reactive with the reference antiserum

The sonicates of all the mycobacteria tested for cross-reactivity were subjected to 2D-IEP employing the reference antiserum against purified cell-mediated immunity target antigen of $M$. tuberculosis strain H37Rv. All the sonicates studied showed a profile similar to that of $M$. tuberculosis strain H37Rv with the $0 \cdot 85-\mathrm{Rf}$ antigen being represented in each of the sonic extracts studied.

\section{Cross-reactivity of 0.85-Rf antigens of different mycobacteria}

The $0 \cdot 85$ - Rf peak-1 antigens of all the mycobacteria tested were individually deposited next to the corresponding antigen of $M$. tuberculosis strain $\mathrm{H} 37 \mathrm{Rv}$ and subjected to rocket immunoelectrophoresis into a gel containing antiserum against the reference antigen, as described previously (Hattikudur and Kamat, 1984). Precipitin peaks so developed were checked for antigenic identity. Irrespective of cross-reactivity in the delayed hypersensitivity response, all the sonicates showed a line of identity between the reference antigen of $M$. tuberculosis strain H37Rv and the corresponding antigen in each of the sonic extracts. Representative profiles of BCG and $M$. phlei are shown in fig. 1 .

Cross-reactivity was also checked by the simple Ouchterlony technique. In a three-way system, as can be seen in fig. 2, crude sonicate of $M$. tuberculosis strain $\mathrm{H} 37 \mathrm{Rv}$, and the purified reference antigen from the same strain were tested against the reference antiserum. The crude sonicate formed four precipitin lines, line 2 of which showed total identity with the single precipitin line formed by the pure reference antigen, thus identifying line 2 as the target antigen of cell-mediated immunity in the crude sonic extract of $M$. tuberculosis strain H37Rv. The sonicate of each mycobacterial strain was then individually tested in a three-well system employing the same 

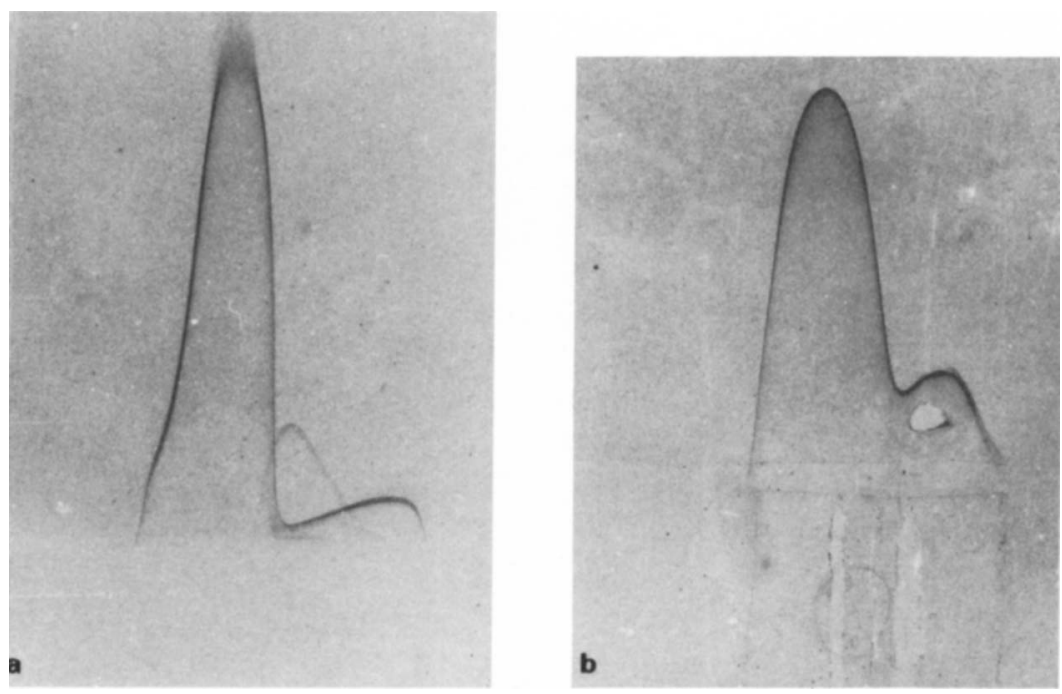

FIG. 1.-Rocket immunoelectrophoresis of purified target antigen of cell-mediated immunity of $M$. tuberculosis strain H37Rv in close vicinity to (a) M. bovis var. BCG, (b) M. phlei. In both, the tall peak belongs to $M$. tuberculosis strain H37Rv.
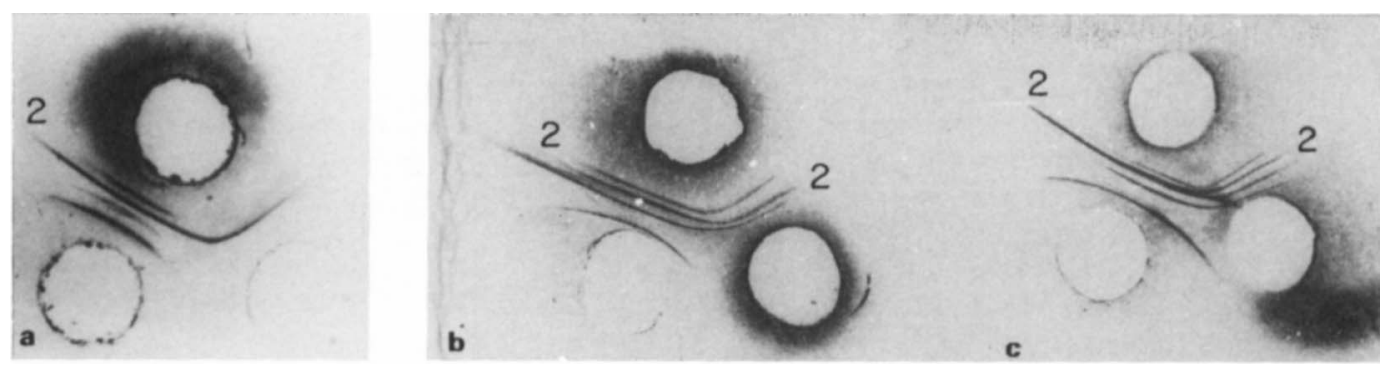

FIG. 2.-Gel diffusion precipitin analysis of sonic extracts of $M$. tuberculosis strain H37Rv, $M$. bovis var. BCG and $M$. phlei. Top well in panels a, b and c contains reference antiserum; left well in panels $\mathrm{a}, \mathrm{b}$ and c contains sonic extract of $M$. tuberculosis strain H37Rv; right well contains (a) purified target antigen of cell mediated immunity of $M$. tuberculosis strain H37Rv, (b) sonic extract of $M$. bovis var. BCG, (c) sonic extract of $M$. phlei. Precipitin-line 2 represents target antigen of cell-mediated immunity in the sonic extracts.

antiserum and $M$. tuberculosis strain H37Rv sonicate in the third well. The precipitin-line 2 of $M$. tuberculosis strain H37Rv showed total antigenic identity with the corresponding antigen of BCG, $M$. marinum and $M$. avium. However, when $M$. phlei was similarly tested, line 2 of $M$. tuberculosis strain H37Rv showed only a partial identity with the corresponding antigen of $M$. phlei, the latter forming a distinct spur over the precipitin line of $M$. phlei. The profiles of BCG and M. phlei are shown in fig. 2.

\section{Discussion}

Sonic extracts of mycobacteria have been reported to contain as many as 40 different antigens (Roberts et al., 1972). Hence it is impossible to study cross-reactivity between organisms without considering the complexity due to multiple antigens. Two 
important questions are (1) are there any unique antigens characteristic of groups and species, and (2) what is the degree of polymorphism of a given well-characterised antigen?

Several mycobacterial proteins such as superoxide dismutase (Kusunose et al., 1980), catalase (Wayne et al., 1976) and other protein antigens such as antigen 5 (Daniel and Janicki, 1978), antigen 21 (Kronvall, Stanford and Walsh, 1976) and antigen 7 of $M$. leprae (Harboe $e t$ al., 1981) have been investigated for polymorphism. Evidence of specificity for individual or a group of two or three mycobacteria has been shown.

Our objective in the study of antigenic polymorphism was more than a study of taxonomic relationships. Because cell-mediated immunity is so important in protection against mycobacterial infection it was of interest to investigate the cross-reactivity of an antigen that is a target of cell-mediated immunity. The reference antigen could not only elicit a delayed hypersensitivity response in mice immunised with live $M$. tuberculosis strain $\mathrm{H} 37 \mathrm{Rv}$, it could also generate activated macrophages via $\mathrm{T}$ cell interaction (Hattikudur and Kamat, 1984). Because this purified antigen was free of carbohydrates and lipids, the reference antiserum raised against it would react against protein antigens only. Thus the serological analysis performed reflects the cross-reactivity of a protein antigen that is a true target of cell-mediated immunity.

Cross-reactivity amongst mycobacteria with delayed hypersensitivity as the test parameter has been extensively studied (Magnusson, 1961) and considerable crossreactivity between $M$. tuberculosis human type and other mycobacteria has been observed. There is a greater degree of cross-reactivity between slow growers as a group than between slow and rapid growers. However, so far, no molecular basis of such cross-reactivity has been established. Antigen 5 (Daniel and Janicki, 1978) is reported to be present only in human and bovine strains. Thus, the basis of cross-reactivity of delayed hypersensitivity responses amongst a number of slowly growing mycobacteria is far from clear. In this respect demonstration of $0.85-\mathrm{Rf}$ target antigen of cell-mediated immunity in all the mycobacteria studied, irrespective of their crossreactivity in a delayed hypersensitivity response, is highly significant.

The antiserum against the reference antigen revealed the presence of a similar antigen in the sonicates of all the mycobacteria studied. It is safe, therefore, to conclude that the antigen has determinants common to all the mycobacteria. This is further confirmed by reactions of identity in the various gel precipitation techniques. The presence of a spur between the reference antigen of $M$. tuberculosis strain H37Rv and the corresponding antigen of $M$. phlei and the lack of a similar spur with the antigen from the sonicates of slowly growing mycobacteria is significant. It suggests that there are two types of antigenic determinants on the same molecule, namely, determinants common to all mycobacteria and determinants shared by slowly growing mycobacteria only. It is possible that species-specific determinants are also present on the same molecule but our antiserum cannot detect such determinants. Antigen 7 (Harboe et al., 1981) and antigen 21 (Kronvall et al., 1976) of $M$. leprae have been reported to have such different determinants on the same molecule.

Stanford et al. (1975) have reported a scheme of classification of mycobacterial antigens. The data in this paper differ from their scheme in some respects. Stanford's group-i antigens are common to all the mycobacteria; his group-ii antigens are common to slow growers only; group-iii antigens are present in rapid growers only and 
group-iv antigens are species specific. These are all separate antigenic molecules whereas in our study the two different classes of antigenic determinants have been demonstrated on the same molecule. It is difficult to explain the observed order of delayed hypersensitivity cross-reactivity amongst mycobacteria (Magnusson, 1961), by the scheme of antigenic classification proposed by Stanford et al. (1976). If the antigen eliciting delayed hypersensitivity had belonged to group $i$, then all mycobacteria would have cross-reacted to the same extent with a given strain; had it been group specific, one would not have seen any cross-reactivity between slow and rapid growers, which is not the case. If the antigen had been species specific there would have been no cross-reactivity at all. The only way that the observed order of cross-reactivity can be fitted into the above scheme of antigenic relationships is to propose that the delayed hypersensitivity response is elicited by multiple antigens. The observed degree of cross-reactivity of a given strain would depend upon the number of antigens it has and the relevant groups they belong to. Alternatively the observed order of cross-reactivity can be explained by the presence of various classes of antigenic determinants on a single molecule, responsible for delayed hypersensitivity - evidence of which has been presented in this study.

In the delayed hypersensitivity response all slow growers cross-reacted strongly with $M$. tuberculosis strain H37Rv and $M$. phlei did not. This suggests that the group-specific determinants on the reference antigen are predominantly involved in cell-mediated immunity, i.e., they constitute the immunodominant group of the antigen in cell-mediated immunity.

\section{REFERENCES}

Daniel T M, Janicki B W 1978 Mycobacterial antigens: A review of their isolation, chemistry, and immunological properties. Microbiological Reviews 42:84-113.

Doub L, Youmans G P 1950 Studies in tuberculosis chemotherapy. 1. Simple primary aromatic amines, in vitro and in vivo. American Review of Tuberculosis 61:407-421.

Freund J E 1967 Modern elementary statistics. 3rd ed, Prentice-Hall Inc. Publications, New Jersey, p 225.

Gray D F, Jennings P A 1955 Allergy in experimental mouse tuberculosis. American Review of Tuberculosis 72:171-195.

Harboe M, Closs O, Reitan L J, Draper P 1981 Demonstration of antibodies reacting with different determinants on Mycobacterium leprae antigen 7. International Journal of Leprosy 49:147-158.

Hattikudur S N, Kamat R S 1984 Characterisation of a target antigen of cell mediated immunity in the sonic extract of $M$. tuberculosis strain H37Rv. Journal of Medical Microbiology $18: 17-25$.

Kronvall G, Stanford J L, Walsh G P 1976 Studies of mycobacterial antigens with special reference to Mycobacterium leprae. Infection and Immunity 13:1132-1138.

Kusunose E, Kusunose M, Ichihara K, Izumi S 1980 Occurrence of superoxide dismutase in Mycobacterium leprae grown in armadillo liver. Journal of General and Applied Microbiology 26:369-372.

Lowry O H, Rosebrough N J, Farr A L, Randall R J 1951 Protein measurement with the folin-phenol reagent. Journal of Biological Chemistry 193:265-275.

Magnusson M 1961 Specificity of mycobacterial sensitins I. Studies in guinea pigs with purified "tuberculin" prepared from mammalian and avian tubercle bacilli, Mycobacterium balnei and other acid-fast bacilli. American Review of Respiratory Diseases 83:57-68.

Ouchterlony O, Nilsson L A 1978 Immunodiffusion and Immunoelectrophoresis. In: Weir D M (ed) Handbook of experimental immunology, 3rd edn. Blackwell Scientific Publications, Oxford, p 19.5 . 
Roberts D B, Wright G L, Affronti L F, Reich M 1972 Characterization and comparison of mycobacterial antigens by two-dimensional immunoelectrophoresis. Infection and Immunity 6:564-573.

Stanford J L, Rook G A W, Convit J, Godal T, Kronvall G, Rees R J W, Walsh G P 1975 Preliminary taxonomic studies on the leprosy bacillus. British Journal of Experimental Pathology 56:579-585.

Wayne L G et al., 1976 Highly reproducible techniques for use in systematic bacteriology in the genus Mycobacterium: tests for niacin and catalase and for resistance to isoniazid thiophene 2-carboxylic acid hydrazide, hydroxylamine, and p-nitrobenzoate. International Journal of Systematic Bacteriology 26:311-318.

World Health Organization 1979 Trial of BCG vaccines in South India for tuberculosis prevention-first report, Tuberculosis prevention trial. Bulletin of the World Health Organization 57:819-827. 